\title{
Introduction to the Processes and Technologies for Small and Large Team Collaboration Minitrack
}

\author{
Douglas C. Derrick \\ University of Nebraska at Omaha \\ dcderrick@,unomaha.edu
}

\author{
Christopher B. R. Diller \\ University of Nebraska at Omaha \\ cdiller@unomaha.edu
}

\author{
Joel H. Helquist \\ Utah Valley University \\ Joel.helquist@uvu.edu
}

\begin{abstract}
Collaboration is a key driver of performance in organizations. The impact of collaboration on organizational performance is more critical than strategic orientation or market and technological turbulence. Yet successful collaboration does not come without difficulty. Groups and teams need to overcome collaboration challenges such as groupthink, dominance, lack of efficiency, and lack of focus. Successful collaboration requires support based on purposeful guidance and interventions to create groups and teams, to design and deploy processes, to design and deploy technology, to support leaders or facilitators, and to improve the efficiency and effectiveness of information processing. The challenge for researchers and practitioners alike is to design sustainable processes and systems within and between organizations that allow people, groups and teams to collaborate successfully. This challenge has many dimensions, including a technical, a behavioral, a social, an emotional, an economical, and a political.

This minitrack provides one of the key international platforms to discuss the following issues:
\end{abstract}

1. Theoretical foundations and design methodologies for collaborative work practices and technologies

2. Processes and tools for establishing and maintaining shared focus and shared mental models over time

3. Processes, technologies, and theoretical breakthroughs to improve and speed up shared sense-making

4. Process and technologies for rapid, collaborative decision-making during crisis

5. Methods and technologies for eliciting and capturing tacit knowledge from experts (i.e., externalization) and sharing / incorporating that knowledge into collaborative efforts (i.e., team internalization)

6. Human collaboration with artificial agents and the evaluation of computer systems as team members, including agent-based support for individual decision makers

7. Automation of collaborative processes and agent- based support for group facilitation

8. Facilitation methods, techniques, patterns, and procedures to improve (a)synchronous collaboration between co-located and distributed people, teams, or groups

9. Assessment models and methods for team collaboration and performance

10. Design, codification and reuse of work practices and pattern languages for group collaboration

This year, we have six great papers that cover a variety of important topics. The first paper explores the process of convergence in crises response, "Convergence on Self-Generated vs. Crowdsourced Ideas in Crisis Response: Comparing Social Exchange Processes and Satisfaction with Process" and provides theoretical understanding this phenomenon. In the next paper, the authors propose a framework for assessing collaborations and prescribing structures and safeguards based on problem complexity and a construct called collaborative distance, "Collaborative Distance: Multi-level Analysis Framework for Recommending Structure and Safeguards". In the third paper of the session, the authors offer a compelling overview of crowdsourcing and IS research, "Theoretical Fashions in Crowdsourcing: A Snapshot of IS Research". For the second session, "Peer-reviewed Brainstorming to Facilitate Large Group Collaboration" proposes some techniques to enhance large group collaboration processes and "Examining Collaboration among Student Teams relying on Web Applications to Coordinate Software Development" gives insight into the collaboration tools for software development. Finally, we conclude with "Collaboration Engineering Methodology: Horizontal Extension to Accommodate Project and Program Concerns", that puts forth recommendations to extend collaboration engineering methodology.

We thank the authors for submitting their work to make this an engaging minitrack. We hope you enjoy the papers and their presentation at the conference and look forward to the collaboration and enlightenment. 